\title{
West Nile Virus (WNV) seroprevalence in a blood donors group of Milan
}

\author{
Giovanna Lunghi ${ }^{2}$, Paolo Gaibani', Caterina Matinato ${ }^{2}$, Anna Orlandi ${ }^{2}$, Antonella Zoccoli', \\ Patrizia Bono', Anna Pierro', Maria Paola Landini', Vittorio Sambri', Erminio Torresani² \\ I Centro di Riferimento Regionale per le emergenze Microbiologiche [CRREM], Unità Operativa di Microbiologia Clinica, \\ Ospedale S.Orsola-Malpighi, Bologna, Italy \\ 2 UO Laboratorio di Analisi Chimico-Cliniche e Microbiologia, Fondazione IRCCS Cà Granda Ospedale Maggiore Policlinico, \\ Milano, Italy
}

Key words: Seroprevalence, West Nile Virus, Blood donors

Sieroprevalenza di West Nile Virus (WNV) in una popolazione di donatori di Milano

\section{SUMMARY}

A seroprevalence study for anti West Nile virus was carried out among 864 healthy blood donors living in the metropolitan area of Milan by using a commercially available ELISA method. In addition, the performance of a novel ELISA assay for WNV antibodies was assessed. The sero-prevalence rate of WNV antibodies was $0.57 \%$ thus showing that WNV is likely circulating also in this up to now unknown area. The overall sensitivity and specificity of the novel ELISA were $99.9 \%$ and $45.4 \%$, respectively, well comparable with that of the chosen reference immunoenzimatic method.

\section{INTRODUZIONE}

Il West Nile virus (WNV) è un Arbovirus appartenente alla famiglia delle Flaviviridae, con un ciclo naturale che coinvolge uccelli (corviviridae) e zanzare (genere Culex). Nella maggior parte dei casi, l'infezione decorre asintomatica con esito di encefalite solo nell' $1 \%$ degli infetti. Tra le vie di trasmissione si riconoscono trasfusioni, trapianto d'organo e via verticale.

Negli ultimi anni la circolazione di WNV è stata descritta ubiquitariamente, con evidenza di epidemie negli Stati Uniti e in Europa $(1,10,11)$.

In Italia la prima epidemia WNV è stata segnalata nel 1998 in Toscana negli equini, il virus è riemerso, poi, nel 2008 -2009 dando origine rispettivamente a 9 e 16 casi di infezione neuro invasiva nell'uomo, in Emilia Romagna e Veneto nella zona del delta del Po $(3,6)$. Come conseguenza di tali ritrovamenti le regioni interessate hanno impostato una strategia di sorveglianza integrata in uomini, animali e vettori per monitorare la circolazione di WNV nel proprio territorio (8).

Tra il 2010 e il 2011 si sono verificati 6 casi di trasmissione di WNV tramite organi trapiantati nonostante la ricerca attiva del DNA del virus tra i donatori nel periodo giugno-novembre (5).

Nel 2011, la presenza di WNV è stata riscontrata, anche in Friuli, in zone naives del sud Italia e Sardegna suggerendo un progressivo allargamente delle zone interessate dal virus (2).

Questo studio è stato intrapreso per valutare la sieroprevalenza di WNV in un'area, quella metropolitana di Milano, dove l'attività virale non era stata documentata precedentemente.

\section{MATERIALI E METODI}

Sono stati esaminati un totale di 864 sieri di donatori afferenti al Centro Trasfusionale della Fondazione Ca' Granda Ospedale Maggiore Policlinico di Milano nel periodo 1 maggio-1 agosto 2011 (età media 45 anni, range $18-65$, ratio maschi/femmine 1.5).

Per lo screening sono stati utilizzati: un nuovo kit commerciale EIA IgG/IgM (DIAPRO Milano) e un kit con performances consolidate, EIA IgG (EuroImmun, Germany). I campioni reattivi sono stati confermati tramite test di microneutralizzazione (MNTA) in accordo con il protocollo standard. I campioni MNTA positivi sono stati valutati mediante EIA IgM, IFA ed Avidity (EuroImmun Germany).

\section{RISULTATI}

Allo screening, 11 campioni sono risultati positivi con il test Diapro e 10 con il test Euroimmun. Tali campioni, confermati tramite microneutralizzazione (MNTA), gold standard per la verifica di specificità degli anticorpi WNV, hanno indicato che solo 5 presentavano azione neutralizzante contro WNV.

La specificità del test Diapro era del $45.5 \%$ mentre quella del test Euroimmun del 54.5\%, la sensi-

\section{Corresponding author: Giovanna Lunghi}

Laboratorio Centrale Analisi Chimico Cliniche e Microbiologia. Fondazione IRCCS Ca' Granda Ospedale Maggiore Policlinico, Milano - Via San Barnaba,8 - Padiglione Devoto - Tel. 0255033457

E-mail: giovanna.lunghi@policlinico.mi.it 
bilità era per entrambi i metodi del 99.9\%, quando comparati con il test MNTA. Il paragone tra i due metodi EIA (Diapro-Euroimmun) indicava un esito concorde nel 91\%, infatti 1 solo campione evidenziava un risultato discordante: positivo Diapro/negativo Euroimmun, confermato negativo dall'MNTA. I 5 campioni positivi alla microneutralizzazione valutati con il test IFA presentavano titoli IgG compresi tra 1/400 e 1/6400, mentre erano tutti negativi per IgM (Tabella 1).

Per stabilire il periodo di infezione i campioni sono stati saggiati con un test di avidità: i titoli sono risultati superiori al $70 \%$, indicando che il contatto con il virus presumibilmente era da datarsi prima della primavera 2011.

\section{CONCLUSIONI}

I risultati del nostro studio confermano la diffusione di WNV nell'area di Milano con una siero prevalenza dello 0.57 tra i donatori di sangue da noi testati. Uno studio simile condotto nel 2009 in
Emilia Romagna, considerata regione endemica per $\mathrm{WNV}$, aveva rivelato una prevalenza dello $0.68 \%$, tra i donatori della provincia di Ferrara (1). In provincia di Rovigo tra i contadini che lavoravano in fattorie con cavalli sieropositivi per WNV la siero prevalenza riportata era dell' $1.56 \%$, mentre era dell' $1.2 \%$, in oltre mille donatori d'organo provenienti da 18 regioni italiane $(3,4,6)$.

Il nostro campione di donatori, tuttavia, rappresentando prevalentemente soggetti maschi tra i 18 e i 65 anni potrebbe sottostimare l'effettiva epidemiologia del virus.

Visti i risultati del test di avidity (>70\%), si può suggerire che WNV circoli almeno dal 2010 tra la popolazione di Milano e che l'attività di sorveglianza stagionale del virus dovrebbe essere estesa a zone più vaste per evitare il rischio di trasmissione tramite donazioni di sangue e d'organo (7).

Il nuovo test EIA saggiato nello screening presenta una sensibilità $(99.9 \%)$ comparabile con i metodi noti e può essere validamente utilizzato in routine.

Tabella I. Riassunto dei test utilizzate per i campioni reattivi.

\begin{tabular}{|c|c|c|}
\hline \multirow[b]{2}{*}{ Campione } & \multicolumn{2}{|c|}{ WNV ELISA IgG } \\
\hline & DiaPro & EUROIMMUN \\
\hline I & Positivo & Positivo \\
\hline 5 & Positivo & Positivo \\
\hline 6 & Positivo & Positivo \\
\hline 15 & Positivo & Positivo \\
\hline 18 & Positivo & Positivo \\
\hline 19 & Positivo & Positivo \\
\hline 22 & Positivo & Positivo \\
\hline 26 & Positivo & Negativo \\
\hline 31 & Positivo & Positivo \\
\hline 33 & Positivo & Positivo \\
\hline 35 & Positivo & Positivo \\
\hline TOT & II & 10 \\
\hline
\end{tabular}

\section{BIBLIOGRAFIA}

1. Angelini P, Tamba M, Finarelli AC, et al. West Nile Virus circulation in Emilia-Romagna, Italy: the integrated surveillance system 2009. Euro Surveill 2010; 15: pii: 19547.

2. Bagnarelli P, Marinelli K, Trotta D, et al. Human case of autochthonous West Nile virus lineage 2 infection in Italy, September 2011. Euro Surveill 2011; 16: pii: 20002.

3. Barzon L, Pacenti M, Cusinato R, et al. Human cases of West Nile Virus infection in north-eastern Italy, 15 June to 15 November 2010. Euro Surveill 2011; 16: pii: 19949.

4. Capobianchi MR, Sambri V, Castilletti C, et al. Italian Transplant Network: Retrospective screening of solid organ donors in Italy, 2009, reveals unpredicted circulation of West Nile virus. Euro Surveil, Aug 26, 2010; 15 (34) pii: 19648.

5. Costa AN, Capobianchi MR, Ippolito G, et al. West Nile virus: the Italian national transplant network reaction to an alert in the north-eastern region, Italy 2011. Euro Surveill 2011; 16: pii: 19991.

\begin{tabular}{|c|c|c|}
\hline $\begin{array}{c}\text { AVIDITY } \\
(\%)\end{array}$ & $\begin{array}{l}\text { WNV IIF } \\
\text { Titolo IgG }\end{array}$ & $\begin{array}{l}\text { MNTA } \\
\text { Titolo }\end{array}$ \\
\hline EUROIMMUN & EUROIMMUN & WNV \\
\hline $\begin{array}{l}\text { Negativo } \\
\geq 70 \%\end{array}$ & $\begin{array}{l}\text { Negativo } \\
\text { I/6400 }\end{array}$ & $\begin{array}{c}\text { Negativo } \\
\text { I/320 }\end{array}$ \\
\hline Negativo & Negativo & Negativo \\
\hline$\geq 90 \%$ & $1 / 1600$ & $1 / 80$ \\
\hline$\geq 90 \%$ & |/400 & $\mathrm{I} / 20$ \\
\hline Negativo & Negativo & Negativo \\
\hline$\geq 70 \%$ & I/6400 & $\mathrm{I} / 40$ \\
\hline Negativo & Negativo & Negativo \\
\hline$\geq 90 \%$ & I/800 & $1 / 80$ \\
\hline Negativo & Negativo & Negativo \\
\hline Negativo & Negativo & Negativo \\
\hline 5 & 5 & 5 \\
\hline
\end{tabular}

6. Cusi MG, Roggi A, Terrosi C, Gori Savellini G, Toti M. Retrospective diagnosis of West Nile virus infection in a patient with meningoencephalitis in Tuscany, Italy. Vector Borne Zoonotic Dis 2011; 11: 1511-2.

7. Fox JL, Hazell SL, Tobler LH, Busch MP. Immunoglobulin $\mathrm{G}$ avidity in differentiation between early and late antibody responses to West Nile virus. Clin Vaccine Immunol 2006; 13: 33-6.

8. Grazzini G, Liumbruno GM, Pupella S, et al. West Nile virus in Italy: a further threat to blood safety, a further challenge to the blood system. Blood Transfus 2008; 6: 235-7.

9. Pierro A, Gaibani P, Manisera C, et al. Seroprevalence of West Nile virus-specific antibodies in a cohort of blood donors in northeastern Italy. Vector Borne Zoonotic Dis 2011; 11: 1605-7.

10. Rizzo C, Vescio F, Declich S, et al. West Nile virus transmission with human cases in Italy, August September 2009. Euro Surveill 2009; 14: pii: 19357.

11. Rossini G, Cavrini F, Pierro A, et al. First human case of West Nile virus neuroinvasive infection in Italy, September 2008 - case report. Euro Surveill 2008; 13: pii: 19002 . 